J. Gynäkol. Endokrinol. AT 2021 · 31:30

https://doi.org/10.1007/s41974-021-00178-8

Online publiziert: 23. Februar 2021

(c) Springer-Verlag GmbH Austria, ein Teil von Springer Nature 2021

\begin{tabular}{c} 
Checc for \\
updates \\
\hline
\end{tabular}

Michael Feichtinger

Wunschbaby Institut Feichtinger, Wien, Österreich

\title{
News-Screen Assistierte Reproduktion
}

der Reduktion der Samenqualität konnte das SARS-CoV-2-Virus in dieser Studie nicht in der Samenflüssigkeit nachgewiesen werden. Allerdings konnten in der Samenflüssigkeit stark erhöhte Entzündungswerte nachgewiesen werden. Ob der beobachtete Effekt dauerhaft oder reversibel ist, ist momentan noch unklar. Wir empfehlen deshalb allen Männern sich, wenn möglich, impfen zu lassen, um kein Risiko hinsichtlich ihrer späteren Zeugungsfähigkeit einzugehen. Männer, die bereits eine Corona-Infektion hatten und einen zukünftigen Kinderwunsch haben, sollten nach einer Ausheilung ein Spermiogramm durchführen lassen.

\section{Originalpublikation}

Mumford SL, Flannagan KS, Radoc JG, et al (2021) Cannabis use while trying to conceive: a prospective cohort study evaluating associations with fecundability, live birth and pregnancy loss. Hum Reprod:deaa355. https:// doi.org/10.1093/humrep/deaa355

\section{Zusammenfassung und Relevanz für die Praxis} Samenqualität dieser Männer. Bei über einem Viertel aller getesteten Männer wurde eine schwere Beeinträchtigung der Samenqualität festgestellt, bei einem Großteil wurde sogar eine Azoospermie festgestellt. Vor allem bei Männern mit einer schweren COVID-19-Infektion war dies besonders ausgeprägt. Viele Erkrankungen, wie beispielsweise eine Grippe, können durch hohe Körpertemperaturen zu einer Beeinträchtigung der Samenqualität führen. Allerdings wiesen die Männer in der vorliegenden Studie 4-8 Wochen nach einer Infektion noch immer stark reduzierte Samenparameter auf, was eine andere Ursache als die erhöhte Körpertemperatur wahrscheinlich macht. Trotz

Allgemein bekannt ist, dass sich Nikotin negativ auf die Samen- und Eizellqualität auswirkt und rauchende Frauen deutlich reduzierte Schwangerschaftsraten haben. Bisher gab es jedoch keine klaren Daten, ob sich ein Cannabiskonsum ebenso auf die Fruchtbarkeit auswirken kann. Laut österreichischem Drogenbericht haben etwa $1 / 4$ aller Österreicher im Laufe ihres Lebens Cannabis konsumiert. Einige westliche Länder (inklusive einiger USBundesstaaten) haben innerhalb der letzten Jahre schrittweise den Gebrauch von Cannabis legalisiert, weshalb auch und besonders junge fruchtbare Frauen zunehmend Cannabis rauchen.
Eine soeben erschienene US-amerikanische Studie an über 1200 Patientinnen mit Kinderwunsch hat anhand von Harnproben Frauen mit Cannabiskonsum identifiziert. Frauen mit Cannabis-Missbrauch in den letzten 12 Monaten hatten eine fast halbierte Wahrscheinlichkeit, schwanger zu werden, obwohl sie häufiger Geschlechtsverkehr hatten als Frauen ohne Cannabiskonsum.

Ein Grund für die erniedrigte Fruchtbarkeit könnte eine direkte Beeinflussung von Cannabis aufden weiblichen Hormonhaushalt sein. So hatten Frauen mit Cannabisgebrauch in den letzten 12 Monaten eine deutlich höhere LH/FSH-Ratio und anovulatorische Zyklen im Vergleich zu Frauen ohne Cannabiskonsum.

$\mathrm{Ob}$ sich der Cannabiskonsum auch langfristig auf die weibliche Fruchtbarkeit auswirkt, bleibt jedoch weiterhin fraglich.

\section{Korrespondenzadresse}

Priv.-Doz. DDr. Michael Feichtinger Wunschbaby Institut Feichtinger Lainzerstraße 6, 1130 Wien,

Österreich

michael.feichtinger@wunschbaby.at
Hinweis des Verlags. Der Verlag bleibt in Hinblick auf geografische Zuordnungen und Gebietsbezeichnungen in veröffentlichten Karten und Institutsadressen neutral. 